\title{
Photometric Redshifts in the Hawaii-Hubble Deep Field-North
}

\author{
G. Yang ${ }^{1}$, Y. Q. Xue ${ }^{1 *}$, B. Luo ${ }^{2}$, W. N. Brandt ${ }^{2}$, D. M. Alexander ${ }^{3}$, \\ F. E. Bauer ${ }^{4}$, W. Cui ${ }^{5}$, X. Kong ${ }^{1}$, B. D. Lehmer ${ }^{6}$, J.-X. Wang ${ }^{1}$, \\ X.-B. Wu ${ }^{7}$, F. Yuan ${ }^{8}$, Y.-F. Yuan ${ }^{1}$ and H. Y. Zhou ${ }^{1}$ \\ ${ }^{1}$ USTC, ${ }^{*}$ xuey@ustc.edu.cn; ${ }^{2}$ PSU; ${ }^{3}$ Durham; ${ }^{4}$ PUC $;{ }^{5}$ Purdue; ${ }^{6}$ JHU; ${ }^{7}$ PKU; ${ }^{8}$ SHAO
}

\begin{abstract}
We derive $z_{\text {phot }}$ for sources in the entire $\left(\sim 0.4 \mathrm{deg}^{2}\right)$ H-HDF-N field with the EAzY code, based on PSF-matched broad-band ( $U$ band to IRAC $4.5 \mu \mathrm{m}$ ) photometry. Our catalog consists of a total of 131,678 sources. We find $\sigma_{\mathrm{NMAD}}=0.029$ for non-X-ray sources. We also classify each object as a star or galaxy through SED fitting. Furthermore, we match our catalog with the 2 Ms CDF-N main X-ray catalog. For the 462 matched non-stellar X-ray sources, we improve their $z_{\text {phot }}$ quality $\left(\sigma_{\mathrm{NMAD}}=0.035\right)$ by adding three additional AGN templates. We make our photometry and $z_{\text {phot }}$ catalog publicly available.
\end{abstract}

Keywords. catalogs — galaxies: distances and redshifts — galaxies: evolution — surveys

There are $>3000 z_{\text {spec }}$ and enormous multiwavelength data in the $\sim 0.4 \operatorname{deg}^{2}$ H-HDF-N field (see Fig.(a)), which covers HDF-N, GOODS-N, CANDELS, and CDF-N. We utilize the EAzY code to derive $z_{\text {phot }}$ with PSF-matched broadband photometry, adopting 8 galaxy templates for non-X-ray sources (see Fig.(b)), 3 additional AGN templates for $\mathrm{X}$-ray sources, and a linear combination of these templates. Our $z_{\text {phot }}$ quality shows overall improvements over relevant previous works, with $\sigma_{\mathrm{NMAD}}=0.029(0.035)$ and an outlier fraction of $5.5 \%(12.5 \%)$ for non-X-ray (X-ray) sources (see Figs.(c-e)). We release photometry and $z_{\text {phot }}$ catalog of 131,678 sources publicly.
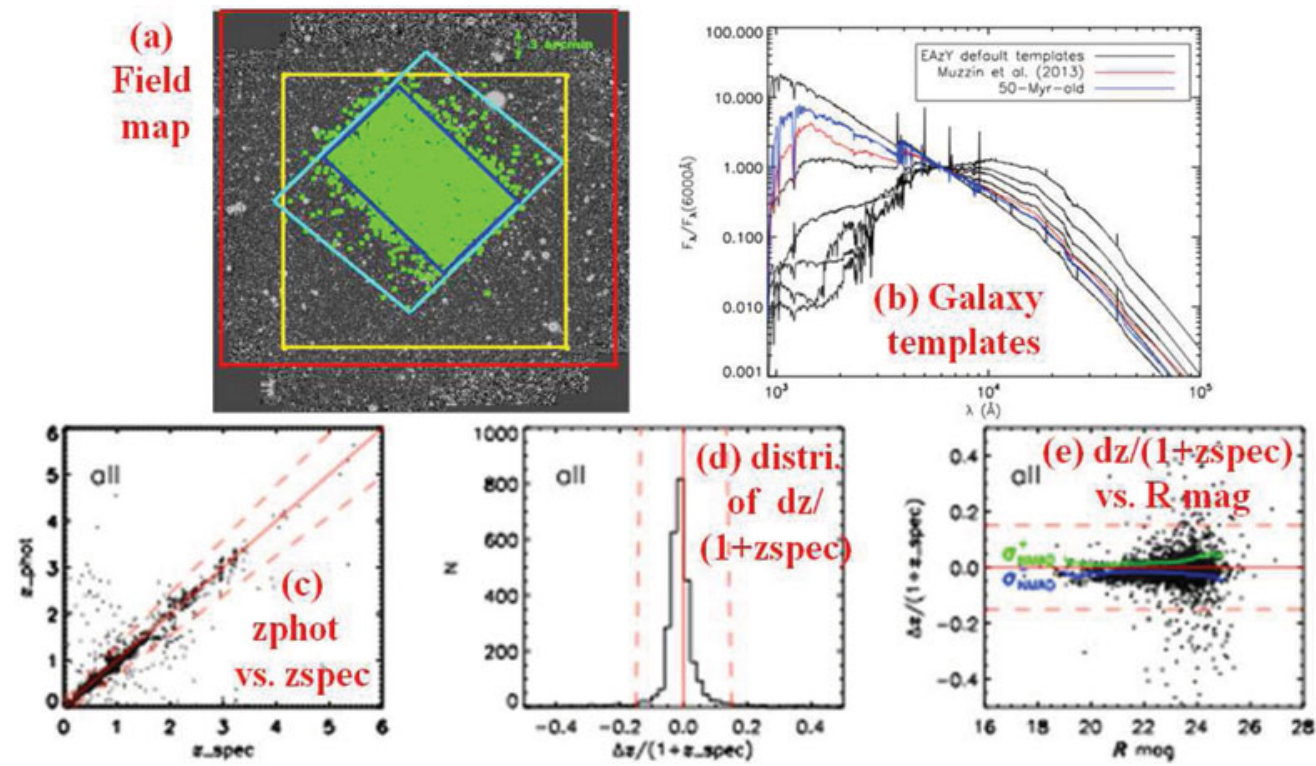